\title{
Coproprevalencia de infestación canina por Echinococcus granulosus en un distrito endémico en hidatidosis en Perú
}

\author{
COPROPREVALENCE OF CANINE INFESTATION BY Echinococcus granulosus IN AN ENDEMIC \\ hidatidosis disctrict in Peru \\ Raúl Montalvo ${ }^{1,2,3}$, Johana Clemente ${ }^{1}$, Lorena Castañeda ${ }^{1}$, Estephany Caro ${ }^{1}$, \\ Yoli Ccente ${ }^{1}$, Mayori Nuñez ${ }^{1}$
}

\section{Resumen}

El objetivo del estudio fue determinar la coproprevalencia de Equinococcus granulosus en canes del distrito rural de San José de Quero, ubicado en la provincia de Concepción, región Junín, en el centro del Perú. El estudio fue observacional de tipo transversal, y se llevó a cabo en 2015 en los tres anexos del distrito. Se recolectaron muestras de heces de 152 canes del mismo número de hogares seleccionados al azar y se procesaron mediante la técnica de ELISA específica de Echinococcus granulosus. El $50 \%$ (Usibamba 61.0\%, Chaquicocha 51.0\% y San José de Quero 41.9\%) de muestras fueron positivas al parásito. Asimismo, se determinó que el promedio de canes por casa fue 2.3 , el $40.9 \%$ de los dueños alimentaba a los canes con vísceras frescas del ganado sacrificado, la proporción de perros que defecan fuera de casa era de $91.3 \%$, y que el $18.4 \%$ refiere haber tenido algún familiar con quiste hidatídico. Se concluye que la infestación canina por E. granulosus es frecuente en el distrito San José de Quero, la cual es facilitada por los hábitos de los dueños y los comportamientos epidemiológicos del can.

Palabras clave: Echinococcus granulosus; coproprevalencia; coproantígeno

\section{AbstraCt}

The objective of this study was to determine the coproprevalence of Equinococcus granulosus in dogs of the rural district of San José de Quero, located in the province of Concepción, Junín region, central Peru. The cross-sectional study was carried out in 2015 in all three annexes of the district. Faecal samples were collected from 152 dogs from the same number of randomly selected households and processed using the Echinococcus

\footnotetext{
${ }^{1}$ Universidad Continental, Huancayo, Perú

${ }^{2}$ Servicio de Enfermedades Infecciosas y Tropicales, Hospital D.A. Carrión, Huancayo, Perú

${ }^{3}$ E-mail: otivo3@hotmail.com
} 
granulosus-specific ELISA technique. The 50\% of samples (Usibamba $61.0 \%$, Chaquicocha $51.0 \%$ and San José de Quero $41.9 \%$ ) were positive to the parasite. Likewise, it was determined that the average number of dogs per house was $2.3,40.9 \%$ of the owners feed the dogs with fresh viscera of slaughtered cattle, the proportion of dogs that defecate outside the house was $91.3 \%$, and that $18.4 \%$ reported having had a family member with a hydatid cyst. It is concluded that canine infestation by E. granulosus is frequent in the district San José de Quero, which is facilitated by the habits of the owners and the epidemiological behaviours of dogs.

Key words: Echinococcus granulosus; coproprevalence; coproantigen

\section{INTRODUCCIÓN}

La equinococosis canina es una enfermedad causada por tenias adultas de Echinococcus granulosus (Boufana et al., 2015). El can se infesta al consumir vísceras infestadas con la forma larvaria del parásito que afecta a ovinos, bovinos y porcinos (Alva et al., 2008). Este agente parasitario está presente en la mayoría de los continentes, desarrollándose especialmente en zonas endémicas donde la ganadería es la principal actividad productiva (Yang et al., 2012). El genotipo 1 (E. granulosus sensu lato) es la forma más prevalente en zonas endémicas de hidatidosis quística humana (Alvarez Rojas et al., 2014).

Esta zoonosis es considerada como una enfermedad desatendida y prevenible (OMS, 2017), prevalente en las regiones alto-andinas (Cucher et al., 2916). Así mismo, se reporta hasta $6.3 \%$ de prevalencia de equinococosis en canes de zonas no endémicas que ingieren vísceras de camales, ya que los animales sacrificados en camales son traídos de zonas endémicas (Reyes et al., 2012).

Amaya et al. (2016) reportaron una prevalencia de infestación canina por $E$. granulosus, determinada mediante copro ELISA de $30.5 \%$ en áreas endémicas y de $11.4 \%$ en zonas no endémicas de Argentina. Así mismo, Merino et al. (2017), utilizando la misma técnica, encontraron $13.8 \%$ de canes infestados y que $27.8 \%$ de los hogares con canes tenían al menos un animal positivo.
En los Andes peruanos, la equinococosis canina es una enfermedad hiperendémica, haciendo que la hidatidosis humana sea un problema de salud relevante en estas zonas. El objetivo de este estudio fue determinar la coproprevalencia de E. granulosus en canes en un distrito de Junín, Perú, con el fin de poder planificar actividades dirigidas al control de esta enfermedad.

\section{Materiales y Métodos}

Se realizó un estudio transversal entre mayo y agosto de 2015 en el distrito de San José de Quero, ubicado en la provincia de Concepción, Junín, Perú. El distrito se encuentra a una altitud de $4100 \mathrm{msnm}$, cuenta con 6452 habitantes y es endémico en hidatidosis quística humana.

Se realizó un muestreo estratificado, dependiendo del número de áreas rurales en los tres anexos del distrito (Usibamba, Chaquicocha, San José de Quero) para estimar el tamaño muestral, considerando una prevalencia de $28 \%$ en canes infestados con E. granulosus en zonas endémicas (AcostaJanett et al. (2014) y de 6\% en zonas no endémicas (Guerra et al., 2015), así como un intervalo de confianza de $95 \%$, poder de $80 \%$ y agregando $20 \%$ por probables pérdidas. El tamaño muestral resultante fue 150 muestras de heces, según el programa estadístico Stata v. 13.1 (Stata Corporation, Texas, EEUU). 
Se recolectaron 152 muestras, correspondiendo 41, 49 y 62 muestras asignadas a los anexos de Usibamba, Chaquicocha y San José de Quero, respectivamente, según el número de habitantes. Para la visita domiciliaria, se seleccionaron en forma aleatoria cuatro hogares por cuadra. Los datos de cada vivienda y de los canes fueron registrados en un cuestionario ad hoc preparado por los autores, que fue evaluado y revisado por dos médicos con especialidad en infectología, un epidemiólogo y dos médicos veterinarios investigadores con registro DYNA - Concytec Perú. Se asignó valores de 0 a 1 punto a cada ítem. Se calculó el valor alfa de Crombach obteniéndose 0.91 , lo cual fue considerando como de muy alta confiabilidad.

El cuestionario incluyó datos de características demográficas de los dueños de los canes, acceso al agua, eliminación de deshechos, crianza de ovejas, sacrificio de ovejas dentro de los hogares, antecedentes de infección hidatídica, contacto con los canes y de la conducta canina como alimentación, permanencia fuera del hogar y factores que influyen en la aparición de E. granulosus. El proceso de recolección de datos y muestra de heces demoró cerca de 20 minutos por hogar. Se enroló un can por vivienda, siendo elegido el de mayor edad en caso de haber varios canes. En casas donde no existían perros se encuestó una vivienda vecina que tuviera canes.

Las muestras de heces de los canes fueron recolectadas mediante hisopado rectal. En los casos donde se encontrase dificultades para la toma de muestras del can elegido, se tomaba la muestra de otro can de la vivienda o se recogían del suelo si es que se podía asegurar que la muestra pertenecía al can elegido para el estudio. Las muestras fueron colocadas en frascos con formol al $10 \%$ y almacenadas a $4{ }^{\circ} \mathrm{C}$ (Moro et al., 1999) hasta su procesamiento. El tiempo entre la recolección de la muestra y el análisis de laboratorio fue de 72 horas en promedio.
Se utilizó la técnica inmunoenzimática (ELISA) comercial para E. granulosus (Cestode Zoonoses Research Group, University of Salford). Las placas se leyeron a $490 \mathrm{~nm}$ con un lector de microplacas Kinetic. Se utilizaron dos controles positivos y dos negativos por placa (Verastegui et al., 1992).

El desarrollo del trabajo fue aprobado por el Comité de Ética del Hospital Daniel Alcides Carrión de Huancayo. Los datos de los participantes fueron registrados mediante códigos, manteniendo la confidencialidad de los mismos.

\section{Resultados}

La encuesta determinó la presencia de 4.5 personas y 2.3 canes por hogar, con una proporción de 0.5 canes por persona. Asimismo, la encuesta indicó que la ganadería y agricultura en conjunto representan el 49.3\% de la principal actividad productiva en cada hogar, que $50.7 \%$ se dedican al comercio, $24 \%$ no tienen acceso al agua potable y $29 \%$ arroja los deshechos al campo. Con relación a la educación, $13 \%$ no tuvieron educación escolarizada y $30 \%$ terminaron su educación primaria. Además, el 18\% de los entrevistados manifestó que al menos algún familiar, no necesariamente intradomiciliario, tuvo hidatidosis quística (Cuadro 1).

El 59\% de los entrevistados crían ovejas, el $41 \%$ alimenta a sus canes con las vísceras de las ovejas que sacrifican en el hogar y el $83 \%$ no desparasita a sus canes. Asimismo, el 33\% de los canes buscan su alimento en las calles. El 43\% de los entrevistados mantiene un contacto cercano con los canes y el $26 \%$ vive cerca de un camal, todo lo cual incrementa el riesgo de infestación con equinococosis.

El anexo de Usibamba tuvo la mayor prevalencia de canes afectados por $E$. granulosus (61.0\%), mientras que los anexos 
Cuadro 1. Características sociodemográficas ( $\mathrm{n}=152$ hogares) de los tres anexos del distrito de San José de Quero, provincia de Concepción, Junín, Perú (2015)

\begin{tabular}{|c|c|c|c|}
\hline \multirow{2}{*}{ Variables } & \multicolumn{3}{|c|}{ Respuestas } \\
\hline & N. ${ }^{\circ}$ & $\%$ & $95 \% \mathrm{CI}$ \\
\hline \multicolumn{4}{|l|}{ Características de los dueños } \\
\hline Personas por hogar ${ }^{1}$ & 4.5 & - & $4.24-4.68$ \\
\hline Sin ningún tipo de educación & 19 & 12.9 & $14.5-17.4$ \\
\hline Actividad ganadera y agrícola & 75 & 49.3 & $40.5-56.9$ \\
\hline Agua potable & 115 & 75.6 & $67.3-81.6$ \\
\hline Desecha la basura detrás de su hogar & 108 & 71.0 & $68.0-82.2$ \\
\hline Alta tasa de contacto con canes & 62 & 43.4 & $34.7-52.1$ \\
\hline Familiar con quiste & 28 & 18.4 & $12.6-25.5$ \\
\hline Número de canes por persona & 0.5 & - & $45.0-61.3$ \\
\hline Camal cerca del hogar & 39 & 26.0 & $19.5-34.1$ \\
\hline \multicolumn{4}{|l|}{ Características de los canes } \\
\hline Edad del can en meses ${ }^{2}$ & 33.7 & - & 28.2- 39.3 \\
\hline Sexo del can: macho & 110 & 73.8 & $70.8-84.5$ \\
\hline Canes por hogar ${ }^{3}$ & 2.3 & - & $2.1-2.9$ \\
\hline Desparasitación anterior & 39 & 25.7 & 18.9- 33.3 \\
\hline Come alimentos de la calle & 50 & 32.9 & $25.5-40.9$ \\
\hline Da las vísceras de ovejas al can & 59 & 41.0 & $32.9-49.1$ \\
\hline El can es callejero & 10 & 6.5 & $3.19-11.7$ \\
\hline Excretan las heces en la calle & 139 & 91.4 & $85.8-95.4$ \\
\hline Duerme en la calle & 52 & 34.2 & $26.7-42.3$ \\
\hline \multicolumn{4}{|l|}{ Características de la oveja } \\
\hline Ha criado alguna vez ovejas & 87 & 59.2 & $50.9-67.1$ \\
\hline Cría ovejas actualmente & 59 & 41.3 & $32.8-49.1$ \\
\hline Numero de ovejas ${ }^{4}$ & 8.5 & - & $5.4-11.7$ \\
\hline Sacrifica ganado en el hogar & 42 & 27.8 & $20.1-34.8$ \\
\hline
\end{tabular}

${ }^{1}$ Rango de personas por hogar: 1-8. ${ }^{2}$ Rango de edad de los canes: 2-168 meses. ${ }^{3}$ Rango de canes por hogar: 0-7. ${ }^{4}$ Rango de ovejas por hogar: 1-45

Cuadro 2. Prevalencia de equinococosis canina en los anexos del distrito de San José de Quero, provincia de Concepción, Junín, Perú (2015)

\begin{tabular}{lcccc}
\hline \multirow{2}{*}{ Anexo } & \multicolumn{2}{c}{ Muestras (n) } & \multicolumn{2}{c}{ Prevalencia } \\
\cline { 2 - 5 } & Total & Positivas & $\%$ & IC 95\% \\
\hline Chaquicocha & 49 & 25 & 51.02 & $0.36-0.66$ \\
San José de Quero & 62 & 26 & 41.94 & $0.33-0.49$ \\
Usibamba & 41 & 25 & 60.98 & $0.52-0.68$ \\
\hline Total & 152 & 76 & 50.0 & $0.41-0.58$ \\
\hline
\end{tabular}


de Chaquicocha y San José de Quero tuvieron el 51.0 y $42.0 \%$ de muestras positivas, respectivamente (Cuadro 2). El 78.3 de los canes muestreados fueron machos.

\section{Discusión}

Los resultados indican una prevalencia de $50 \%$ de equinococosis canina y la presencia de 0.5 canes por persona, lo cual permite extrapolar la existencia de 1709 canes infestados por E. ganulosus en el distrito San José de Quero. No obstante, esta prevalencia es relativamente baja en comparación a estudios realizados en otras áreas rurales de Perú, como en el norte de Lima y en la zona de Vichaycocha (Andes de Lima), donde se reportaron 82 y $89 \%$ de canes infestados, respectivamente, usando el test coproantigeno mediante ELISA (Moro et al., 1999), aunque similar al 51\% de prevalencia en la sierra de Lima (Moro et al., 2005).

La mayor presencia de canes machos $(78.3 \%)$ es un claro indicativo del favoritismo de los dueños pues ayudan en el cuidado del ganado. Esto incrementa el riego de equinococosis en los perros, toda vez que Moro et al. (2005) señala que los canes machos tienen 1.5 veces más riesgo de infestación que las hembras. Por otro lado, solo el $25.7 \%$ de los canes fueron desparasitados anteriormente, valor inferior a la desparasitación reportada en zonas urbanas del departamento costero de Ica (36.7\%) (Cabrera et al., 2013).

En un estudio sobre factores de riesgo de hidatidosis en la población humana en un distrito minero de Cerro de Pasco, Perú, se señala que el $7.3 \%$ de los pobladores de la zona rural no había recibo instrucción en comparación con el $4.9 \%$ de analfabetos en la zona urbana (Núñez et al., 2001). En el presente estudio, el porcentaje de analfabetismo fue mayor (12.9\%), probablemente debido a que la actividad ganadera y agrícola son la mayor fuente de su ingreso económico.
El 71.15\% de las personas desechan la basura detrás de los hogares, contaminando al ambiente e incrementando el riesgo de propagación de enfermedades. Cabrera et al. (2013), asimismo, reportan para la zona endémica de hidatidosis/equinococosis de Ica, Perú, que el $29.1 \%$ de los pobladores desechan la basura fuera del hogar. La Municipalidad del distrito San José de Quero recoge semanalmente los desechos de cada anexo por medio de un carro recolector.

El hábito arraigado de ofrecer a los canes las vísceras de los animales sacrificados (Buishi et al., 2005), explica la alta prevalencia de equinococosis en los canes. Es evidente la mayor probabilidad de infección por E. granulosus en canes que están en contacto directo con el ganado y que ingieren restos de vísceras (Moro et al., 1999).

El acceso de canes a los despojos de vísceras del ganado como son los camales y lugares de beneficio de animales y el acceso a las áreas de cría de ganado son condiciones favorables para la diseminación de la equinococosis (Otero-Abad y Torgerson, 2013), al igual que la vagancia y la deposición en la calle, características que fueron comunes en este estudio. Los canes libres presentan mayor riesgo de infestarse en comparación a los canes que viven al interior de los hogares (Parada et al., 1995: Buishi et al., 2005, 2006). Del mismo modo, los canes callejeros muestran mayor intensidad de infección en comparación con los canes caseros (Inangolet et al., 2010).

La infestación canina por $E$. granulosus representa un serio problema de salud pública en las zonas alto-andinas y ganadera; de allí que debería realizarse un mapeo epidemiológico de lugares específicos para intervenciones dirigidas.

\section{Agradecimientos}

Se agradece por su colaboración a los médicos serumnistas Sofía Olivera Núñez y Juan José Flores. 


\section{Literatura Citada}

1. Acosta-Jamett G, Weitzel T, Boufana B, Adones C, Bahamonde A, Abarca K, Craig P, et al. 2014. Prevalence and risk factors for echinococcal infection in a rural area of northern Chile: a household-based cross-sectional study. PLoS Neg1 Trop Dis 8(8): e3090. [Internet]. Disponible en: https://doi.org/ 10.1371/journal.pntd.0003090

2. Alva P, Cornejo W, Sevilla C, Huiza A. 2008. Encuesta serológica para hidatidosis humana por la prueba de doble difusión Arco 5 en la provincia de Chupaca, Junín, Perú. Rev Peru Med Exp Salud Pública 25: 149-152. doi: 10.17843/rpmesp.2008.251.1240

3. Alvarez Rojas CA, Romig T, Lightowlers MW. 2014. Echinococcus granulosus sensu lato genotypes infecting humans - review of current knowledge. Int J Parasitol 44: 9-18. doi: 10.1016/j.ijpara.2013.08.008

4. Amaya JC, Moreno N, Salmaso N, Bazán E, Ricoy G, Córdoba P, Santillán G 2016. Estudio de infestación de caninos con Echinococcus granulosus en la provincia de La Rioja, Argentina. Rev Argent Microbiol 48: 3842. doi: 10.1016/j.ram.2015.11.003

5. Boufana B, Lett W, Lahmar S, Griffiths A, Jenkins DJ, Buishi I, Engliez SA, et al. 2015. Canine echinococcosis: genetic diversity of Echinococcus granulosus sensu stricto (s.s.) from definitive hosts. J Helminthol 89: 689-698. doi: 10.1017/S0022149X15000395

6. Buishi I, Njoroge E, Zeyhle E, Rogan MT, Craig PS. 2006. Canine echinococcosis in Turkana (northwestern Kenya): a coproantigen survey in the previous hydatid-control area and an analysis of risk factors. Ann Trop Med Parasitol 100: 601-610. doi: 10.1179/ 136485906X118503
7. Buishi I, Walters T, Guildea Z, Craig P, Palmer S. 2005. Reemergence of canine Echinococcus granulosus infection, Wales. Emerg Infect Dis 11: 568-571. doi: 10.3201/eid1104.040178

8. Buishi IE, Njoroge EM, Bouamra O, Craig PS. 2005. Canine echinococcosis in northwest Libya: assessment of coproantigen ELISA, and a survey of infection with analysis of risk-factors. Vet Parasitol 130: 223-232. doi: 10.1016/ j.vetpar.2005.03.004

9. Cabrera $R$, Talavera E, TrilloAltamirano M. 2013. Conocimientos, actitudes y prácticas de los matarifes acerca de la hidatidosis/equinococosis, en dos zonas urbanas del Departamento de Ica, Perú. An Fac Med Lima 66: $203-$ 211. doi: 10.15381/anales.v66i3.1340

10. Cucher MA, Macchiaroli N, Baldi G, Camicia F, Prada L, Maldonado L, Avila HG, et al. 2016. Cystic echinococcosis in South America: systematic review of species and genotypes of Echinococcus granulosus sensu lato in humans and natural domestic hosts. Trop Med Int Health 21: 166-175. doi: 10.1111/tmi.12647

11. Guerra LM, Ramírez MC. 2015. Hidatidosis humana en el Perú. Apunt Cienc Soc 5: 94-101. doi: 10.18259/ acs. 2015015

12. Inangolet FO, Biffa D, Opuda-Asibo J, Oloya J, Skjerve E. 2010. Distribution and intensity of Echinococcus granulosus infections in dogs in Moroto District, Uganda. Trop Anim Health Prod 42: 1451-1457. doi: 10.1007/ s11250-010-9574-6

13. Merino V, Falcón $N$, Morel $N$, González G. 2017. Detección de coproantígenos de Echinococcus granulosus en canes de trabajadores de camales y comercializadores de vísceras en Lima metropolitana. Rev Panam Salud Publica 41: e10. [Internet]. Disponible en: http://iris.paho.org/xmlui/ handle/123456789/33840 
14. Moro PL, Bonifacio N, Gilman RH, Lopera L, Silva B, Takumoto R, Verástegui M, et al. 1999. Field diagnosis of Echinococcus granulosus infection among intermediate and definitive hosts in an endemic focus of human cystic echinococcosis. Trans R Soc Trop Med Hyg 93: 611-615. doi: 10.1016/S0035-9203(99)90068-8

15. Moro PL, Lopera L, Bonifacio N, Gonzales A, Gilman RH, Moro MH. 2005. Risk factors for canine echinococcosis in an endemic area of Peru. Vet Parasitol 130: 99-104. doi: 10.1016/j.vetpar.2005.03.015

16. Núñez, E, Calero D, Estares L, Morales A. 2003. Prevalencia y factores de riesgo de hidatidosis en población general del distrito de Ninacaca-Pasco, Perú. An Fac Med Lima 64: 34-42. doi: 10.15381/anales.v64i1.1419

17. [OMS] Organización Mundial de la Salud. 2017. Equinococosis. [Internet]. Disponible en: http://www.who.int/ mediacentre/factsheets/fs377/es/

18. Otero-Abad B, Torgerson PR. 2013. A systematic review of the epidemiology of echinococcosis in domestic and wild animals. PLoS Negl Trop Dis 7(6): e2249.
[Internet]. Disponible en: https://doi.org/ 10.1371/journal.pntd.0002249

19. Parada L, Cabrera P, Burges C, Acuña A, Barcelona $C$, Laurenson $M K$, Gulland FM, Agulla J, et al. 1995. Echinococcus granulosus infections of dogs in the Durazno region of Uruguay. Vet Rec 136: 389-391.

20. Reyes MM, Taramona CP, SaireMendoza M, Gavidia CM, Barron E, Boufana B, Craig PS, et al. 2012. Human and canine echinococcosis infection in informal, unlicensed abattoirs in Lima, Peru. PLoS Negl Trop Dis 6(4): e1462. doi: 10.1371/journal.pntd.0001462

21. Yang YR, Clements ACA, Gray DJ, Atkinson J-AM, Williams GM, Barnes TS, McManus D. 2012. Impact of anthropogenic and natural environmental changes on Echinococcus transmission in Ningxia Hui Autonomous Region, the People's Republic of China. Parasit Vectors 5: 146. doi: 10.1186/1756-33055-146

22. Verastegui M, Moro P, Guevara A, Rodriguez T, Miranda E, Gilman RH. 1992. Enzyme-linked immunoelectrotransfer blot test for diagnosis of human hydatid disease. J Clin Microbiol 30: 1557-1561. 